\title{
NORMAL FORMS OF INVARIANT VECTOR FIELDS UNDER A FINITE GROUP ACTION
}

\author{
FEDERICO SÁNCHEZ-BRINGAS
}

\begin{abstract}
Let $\Gamma$ be a finite subgroup of $G L(n, \mathbb{C})$. This subgroup acts on the space of germs of holomorphic vector frelds vanishing at the origin in $\mathbb{C}^{n}$ and on the group of germs of holomorphic diffeomorphisms of $\left(\mathbb{C}^{n}, 0\right)$. We prove a theorem of invariant conjugacy to a normal form and linearization for the subspace of invariant germs of holomorphic vector fields and we give a description of this type of normal forms in dimension $n=2$.
\end{abstract}

\section{Introduction}

The goal of this paper is to show that the classic theorems of PoincareDulac [DU] and Siegel [SI] of conjugacy to a normal form and linearization of germs of holomorphic vector fields at $0 \in \mathbf{C}^{n}$ hold for the quotient space $\mathrm{C}^{n} / \Gamma$, where $\Gamma$ is a finite subgroup of $G L(n, \mathrm{C})$. In this situation we consider the germs of holomorphic vector fields and the germs of conjugating diffeomorphism of $\mathbf{C}^{n}$ invariant by the action of the subgroup.

It is well known that $\mathbf{C}^{n} / \Gamma$ has the structure of an algebraic variety and furthermore any variety which is the quotient of a finite group of local diffeomorphisms of $\mathrm{C}^{n}$ is of this form (in a specific system of coordinates) $[\mathbf{C A}]$, then we obtain here results for conjugacies to normal forms and linearizations of germs of holomorphic vector fields in this kind of algebraic varieties.

In a different context, like bifurcation theory, sometimes conjugacy to a normal form of germs of holomorphic vector fields which preserves symmetries are needed, this results can also be applied.

In the first section we prove the main theorem using the algebraic approach developed in $[\mathbf{C H}]$. In the second section we analyse carefully the case $\mathrm{C}^{2} / \Gamma$ and we give a description of normal forms.

Finally we wish to thank Xavier Gomez-Mont for his helpful comments and remarks concerning this work. 


\section{Invariant conjugacy to a normal form and linearization in $\mathrm{C}^{n}$}

Let $\chi\left(\mathrm{C}^{n}, 0\right)$ be the space of germs of holomorphic vector fields at $0 \in \mathbf{C}^{n}$ vanishing at the origin. Let $\Gamma$ be a finite subgroup of $G L(n, \mathrm{C})$ which acts naturally on $\chi\left(\mathrm{C}^{n}, 0\right)$, we say that $X$ is invariant if it is invariant by this action, namely if for all $\gamma \in \Gamma, d \gamma^{-1}(X(\gamma(z))=X(z)$. Let $\chi\left(\mathrm{C}^{n} / \Gamma, 0\right)$ be the subspace of invariant elements of $\chi\left(\mathrm{C}^{n}, 0\right)$.

Given $X \in \chi\left(\mathbf{C}^{n}, 0\right)$ denote by $X_{1}$ its linear part, $d X(0)$ and suppose it belongs to $G L(n, \mathrm{C})$. Let $S$ be the semisimple part of $X_{1}$, we say $X$ is a normal form if $L_{S} X=0$, where $L_{S}$ is the Lie derivative of $S$.

$X_{1}$ is said to be resonant if its eigenvalues satisfy a relation (resonance) like this:

$$
\lambda_{u}=\sum_{1}^{n} m_{i} \lambda_{i}, u=1, \ldots, n,\left(m_{1}, \ldots, m_{n}\right) \in \mathrm{N}^{n}, \sum_{1}^{n} m_{i} \geq 2 .
$$

The vector field $z_{1}^{m_{1}} \ldots z_{n_{2}}^{m_{n}} \frac{\partial}{\partial z_{1}}, u=1, \ldots, n$ is the monomial vector field associated to this resonance.

Suppose that $X \in \chi\left(\mathrm{C}^{n}, 0\right)$ is a normal form and the coordinates of $\mathrm{C}^{n}$ are given by a basis of eigenvectors of the semisimple part $S$ of $X_{1}$. Then condition $L_{S} X=0$ implies that $X-X_{1}$ is a sum of resonant monomial vector fields.

Let Diff $\left(\mathbf{C}^{n}, 0\right)$ be the group of germs of holomorphic diffeormorphisms which fix the origin of $\mathrm{C}^{n}, \Gamma$ acts by conjugation here. We say that $\phi \in \operatorname{Diff}\left(\mathbf{C}^{n}, 0\right)$ is invariant if it is invariant by this action, namely if for all $\gamma \in \Gamma, \gamma^{-1} \phi \gamma=\phi$. Denote by $\operatorname{Diff}\left(\mathrm{C}^{n} / \Gamma, 0\right)$ the group of invariant clements of $\operatorname{Diff}\left(\mathrm{C}^{\mathrm{n}}, 0\right)$.

For any $X, Y \in \chi\left(\mathbf{C}^{n}, 0\right)$ we say $X$ is conjugate to $Y$ if there is a $\phi \in \operatorname{Diff}\left(\mathbf{C}^{n}, 0\right)$ such that $\phi^{*} X=Y$, where $\phi^{*} X=d \phi^{-1} X \phi$. When $Y$ is the linear part of $X$ we say $X$ is linearizable. If $X$ and $Y$ are invariant, the conjugacy (linearization) is called invariant.

Theorem 1. Let $\Gamma$ be a finite subgroup of $G L(n, \mathrm{C})$ and $X \in \chi\left(\mathbf{C}^{n} / \Gamma, 0\right)$. Suppose the linear part $X_{1}$ of $X$ is invertible. Then:

1.1. $X$ is invariantly conjugate, possibly formally to a normal form. If $X_{1}$ is non-resonant then this conjugacy is an invariant linearization.

1.2. If $X$ is holomorphically conjugate to a normal form, then it can be conjugate in an invariant holomorphic way.

The proof of this theorem is a consequence of the following lemma. Let $\mathcal{O}_{\mathrm{C}^{n}, 0}$ be the algebra of germs of holomorphic functions at $0 \in \mathrm{C}^{n}$ and 
$m=\left\{f \in \mathcal{O}_{\mathbb{C}^{n}, 0} ; f(0)=0\right\}$ its maximal ideal. For each non-negative integer $k$ denote $\mathcal{J}_{\mathrm{C}^{n}, 0}^{k}=\mathcal{O}_{\mathrm{C}^{n}, 0} / m^{k}$ the algebra of finite dimension of $k$-jets of holomorphic functions. The element $X \in \chi\left(\mathrm{C}^{n}, 0\right)$ defines a derivation $X^{*}$ of $\mathcal{O}_{\mathbf{C}^{n}, 0}, X^{*} f=L_{X} f$ and in a natural way the $k$-jet of $X$ determines a derivation $X_{k}^{*}$ of $\mathcal{J}_{\mathrm{C}^{n}, 0}^{k}$, then $X_{k}^{*}$ has a canonical decomposition: $X_{k}^{*}=S_{k}^{*}+N_{k}^{*}$, where $S_{k}^{*}$ is the semisimple part and $N_{k}^{*}$ is the nilpotent part. A remarkable fact proved in $[\mathrm{CH}]$ is that $S_{k}^{*}$ is a derivation.

In a similar way, we denote by Diff $_{k}\left(\mathbf{C}^{n}, 0\right)$ the group of $k$-jets of germs of holomorphic diffeomorphisms of $\mathrm{C}^{n}$. The definition of conjugacy to a normal form (linearization) is extended in a natural way to the space of $k$-jets of germs of vector fields, $\chi^{k}\left(\mathbf{C}^{n}, 0\right)$.

Lemma 2. Let $\Gamma$ be a finite subgroup of $G L(n, C), k$ a non-negative integer and $X_{k} \in \chi^{k}\left(\mathrm{C}^{n} / \Gamma, 0\right)$ then

2.1. The semisimple part $S_{k}^{*}$ of $X_{k}^{*}$ is invariant.

2.2. $S_{k}^{*}$ is invariant linearizable if $X_{1}$ is invertible.

Proof: 2.1. On one hand we have the following fact $[\mathrm{Hu}]$ :

Let $V$ be a $\mathrm{C}$-vector space of finite dimension and $T$ an endomorphism of $V$. Then the semisimple part of $T$ has a polynomial expression in $T, p(T)$ with coefficients in $\mathbf{C}$. On the other hand, as $X$ is invariant and $\gamma \in \Gamma$ is linear we have $d \gamma^{-1} X_{\gamma}=\gamma^{-1} X_{\gamma}=X$ then for any nonnegative integer $k, \gamma^{-1} X_{k}^{*} \gamma=X_{k}^{*}$ and $\gamma^{-1} X_{k}^{*} \circ \cdots \circ X_{k}^{*} \gamma=X_{k}^{*} \circ \cdots \circ X_{k}^{*}$ then any polynomial expression in $X_{k}^{*}$ with coefficients in $\mathrm{C}$ is invariant.

2.2. Let $\phi_{k} \in \operatorname{Diff}_{k}\left(\mathbf{C}^{n}, 0\right)$ be the Poincaré-Dulac diffeomorphism which exists because $X_{1}$ is invertible. $\phi_{k}$ is tangent $((k-1)$-order $)$ to the identity diffeomorphism, and linearizes the semisimple part of $X_{k}^{*}$. Define the average $\ddot{\phi}_{k}=|\Gamma|^{-1} \sum_{\gamma \in \Gamma} \gamma^{-1} \phi_{k} \gamma$.

$\tilde{\phi}_{k}$ is invariant and tangent $((k-1)$-order $)$ to the identity diffeomorphism. Besides

$$
\begin{aligned}
\left(|\Gamma|^{-1} \sum_{\gamma \in \Gamma} \gamma^{-1} \phi_{k} \gamma\right)^{*}\left(S_{k}^{*}\right) & =|\Gamma|^{-1} \sum_{\gamma \in \Gamma}\left(\gamma^{-1} \phi_{k}^{-1} \gamma\right)_{*} S_{k}^{*}\left(\gamma^{-1} \phi_{k} \gamma\right) \\
& =|\Gamma|^{-1} \sum_{\gamma \in \Gamma} \gamma S_{1}^{*} \gamma^{-1} \\
& =S_{1}^{*}
\end{aligned}
$$

where $S_{1}^{*}$ is the linear part of $S_{k}^{*}$ which is invariant because of $S_{k}^{*}$. 
Proof of the theorem: 1.1. $L_{S_{1}} X_{k}=0$ if and only if $0=\left(L_{S_{1}} X_{k}\right)^{*}=$ $S_{1}^{*} X_{k}^{*}-X_{k}^{*} S_{1}^{*}$, then the canonical decomposition of $X_{k}^{*}$ implies that conjugating to a normal form in $\chi^{k}\left(\mathrm{C}^{n}, 0\right)$ is equivalent to linearizing the semisimple part $S_{k}^{*}$. Now let $\tilde{\phi}_{k}$ be like in lemma 2. Remark that if we write

$$
\begin{aligned}
\tilde{\phi}_{l} \circ \cdots \circ \tilde{\phi}_{2} & =\mathrm{Id}+\phi^{2}+\cdots+\phi^{l}+\ldots \text { then } \\
\tilde{\phi}_{t+1} \circ \tilde{\phi}_{l} \circ \cdots \circ \tilde{\phi}_{2} & =\left(\mathrm{Id}+\phi^{2}+\cdots+\phi^{l}+\phi^{i+1}+\ldots\right)+\phi^{l+1}(\mathrm{Id}+\ldots)+\ldots \\
& =\mathrm{Id}+\phi^{2}+\cdots+\phi^{l}+\ldots
\end{aligned}
$$

so this two compositions have the same $l$-jet and $\tilde{\phi}_{k} \circ \cdots \circ \tilde{\phi}_{2}$ conjugates invariantly $X_{k}$ to a normal form because as we showed, this diffeomorphism linearizes $S_{k}^{*}$. Finally the $\operatorname{limit}_{k \rightarrow \infty}\left(\tilde{\phi}_{k} \circ \cdots \circ \tilde{\phi}_{2}\right)$ defines a diffeomorphism $\tilde{\phi}$, eventually formal which conjugates invariantly $X$ to a normal form.

1.2. Let $\phi$ be the holomorphic conjugacy (in any system of coordinates) then a similar argument as in 1 implies that $|\Gamma|^{-1} \sum_{\gamma \in \Gamma} \gamma^{-1} \phi \gamma \in$ $\operatorname{Diff}\left(\mathbf{C}^{n}, 0\right)$ conjugates $X$ holomorphically and invariantly to the respective normal form.

\section{Description in $\mathrm{C}^{2}$ : Invariant normal forms}

Let $X_{1}$ be a linear vector field in $\mathbf{C}^{2}$ with eigenvalues $\lambda_{1}, \lambda_{2}$. Choose a base of $\mathbf{C}^{2},\left\{e_{1}, e_{2}\right\}$ of eigenvectors of $S$, the semisimple part of $X_{1}$. We say that $X_{1}$ belongs to the Poincaré domain if 0 is not in the segment $\left[\lambda_{1}, \lambda_{2}\right]$. Otherwise we say $X_{1}$ belongs to the Siegel domain. The eigenvalues $\lambda_{1}, \lambda_{2}$ are of type $(C, \nu), C, \nu \geq 0$ if: $\left|\lambda_{u}-m_{1} \lambda_{1}-m_{2} \lambda_{2}\right| \geq$ $C\left(\left|m_{1}\right|+\left|m_{2}\right|\right)^{-\nu}$ for all $\left(m_{1}, m_{2}\right) \in\left(\mathbf{N}^{2}\right)^{*}, u=1,2$.

Before applying theorem 1 in this context we point out that conditions to $X$ be conjugate holomorphically to a normal form have been established in [DU] if $X_{1}$ is in the Poincare domain and in [SI] if $X_{1}$ belongs to the Siegel domain.

Theorem 3. Let $X_{1}$ be the linear part of $X \in \chi\left(\mathbf{C}^{n} / \Gamma, 0\right)$. Let $\lambda_{1}, \lambda_{2}$ be the eigenvalues of $X_{1}$.

3.1. If $X_{1}$ is not resonant, then $X$ is linearizable in a holomorphic invariant way in the following cases:

i) $X_{1}$ belongs to the Poincaré domain.

ii) $X_{1}$ belongs to the Siegel domain and $\lambda_{1}, \lambda_{2}$ are of type $(C, \nu)$ for some $C, \nu \geq 0$. 
3.2. If $X_{1}$ is resonant, then $X$ is conjugate in a holomorphic invariant way to a normal form in the following cases:

i) $X_{1}$ belongs to the Poincaré domain.

ii) $X_{1}$ belongs to the Siegel domain and the normal form is colinear to $X_{1}$.

Remark. There exist cases where the invariant conjugacy to a normal form is only formal. For example if $\Gamma$ is a diagonal group (i.e. each of its elements are diagonal) we are going to show there are normal forms with linear part in the Siegel domain which do not verify condition 2,ii). In this case the conjugating diffeomorphism $\phi$ may be divergent because one of its coordinate functions can have coefficients which grow like the Euler function, $[\mathbf{B r}]$ :

$$
\eta(z)=\sum_{\delta=1}^{\infty}(\delta-1) ! z^{\delta} .
$$

We express the invariance condition in $\chi\left(\mathbf{C}^{2}, 0\right)$ with an average morphism of the group action. Let $\Pi: \chi\left(\mathbf{C}^{2}, 0\right) \rightarrow \chi\left(\mathbf{C}^{2} / \Gamma, 0\right)$ be the morphism of $\mathbf{C}$-vectorial spaces defined by $\Pi(X)=|\Gamma|^{-1} \sum_{\gamma \in \Gamma} \gamma^{*} X$. Then $X$ is invariant if and only if $\Pi(X)=X$.

There are two different cases for the family of finite subgroups of $G L(2, \mathbf{C})$ :

i) If $\Gamma$ is diagonal, the monomial vector fields are eigenvectors of $\Pi$ and $\Pi(X)=0$ if $X$ is not invariant.

ii) If $\Gamma$ is not diagonalizable the eigenvectors of $\Pi$ are not monomials and $\Pi$ does not vanish monomials.

Let us regard first the case of diagonalizable groups.

Proposition 4. If $\Gamma$ is not diagonalizable and $X_{1}$ is a linear vector feld, then $\Pi\left(X_{1}\right)=X_{1}$ if and only if $X_{1}=\lambda\left(z_{1}, z_{2}\right)$.

Proof: Suppose $X_{1}$ is given in its Jordan canonical form. If $X_{1}$ has different eigenvalues the condition $X_{1} \gamma=\gamma X_{1}$ implies $\gamma$ is diagonal, therefore $\Gamma$ must be diagonal.

If

$$
X_{1}=\left(\begin{array}{cc}
\lambda & 1 \\
0 & \lambda
\end{array}\right)
$$

$X_{1} \gamma=\gamma X_{1}$ implies

$$
\gamma=\left(\begin{array}{cc}
\mu & b \\
0 & \mu
\end{array}\right)
$$

but $\Gamma$ is finite then $\gamma^{n}=\mathrm{Id}$ and $b$ must be 0 . 
Remark. This proposition implies that for non diagonalizable groups our theorem is a linearizing theorem illustrated by the following example: Let $\Gamma$ be the binary dihedral group generated by

$$
\gamma=\left(\begin{array}{cc}
i & 0 \\
0 & -i
\end{array}\right) \text { and } j=\left(\begin{array}{rr}
0 & -1 \\
1 & 0
\end{array}\right)
$$

The vector field $X\left(z_{1}, z_{2}\right)=\left(z_{1}, z_{2}\right)+\left(z_{1}^{5} ; z_{2}^{5}\right)$ is not a multiple of the radial vector field which belongs to $\chi\left(\mathbf{C}^{2} / \Gamma, 0\right)$.

Suppose now the group is diagonal. In order to simplify the description of invariant normal forms we will suppose $\Gamma$ is cyclic and generated by

$$
\left(\begin{array}{cc}
e^{\left(2 \pi i_{1} \sqrt{-1}\right) / n_{1}} & 0 \\
0 & e^{\left(2 \pi i_{2} \sqrt{-1}\right) / \pi_{2}}
\end{array}\right)
$$

$n_{u} \in \mathbf{N}, l_{u} \in \mathbf{Z},\left(l_{u}, n_{u}\right)=1, u=1,2$.

If $X\left(z_{1}, z_{2}\right)=\sum_{u=1,2}\left(\sum_{i, j=0}^{\infty} c_{i j}^{u} z_{1}^{i} z_{2}^{j}\right) e_{u}$, the equivariance condition is imposed independently for monomial vector fields. The next proposition describes invariant vector fields.

Proposition 5. Let $n$ be the least common multiple of $n_{1}, n_{2}$ and $\eta_{u}=n l_{u} / n_{u}$. The monomial vector field $z_{u}\left(z_{u}^{i} z_{v}^{j}\right) e_{u}, u=1,2, u \neq v, i \geq$ $-1, j \geq 0, i+j \geq 0$ is invariont if and only if $\eta_{u} i=-\eta_{v} j(\bmod n)$.

Proof: Suppose

$$
\gamma=\left(\begin{array}{cc}
\gamma_{1} & 0 \\
0 & \gamma_{2}
\end{array}\right)
$$

belongs to $\Gamma$ then $\Pi\left(z_{1}^{i} z_{2}^{j} e_{u}\right)=\left(1 / n \sum_{\gamma \in \Gamma} \gamma_{u}^{-1} \gamma_{1}^{i} \gamma_{2}^{j}\right) z_{1}^{i} z_{2}^{j} e_{u}$, therefore $z_{1}^{i} z_{2}^{j} e_{u}$ is invariant if and only if $n^{1} \sum_{\gamma \in \Gamma} \gamma_{u}^{-1} \gamma_{1}^{i} \gamma_{2}^{j}=1$. If $u=1$, $\sum_{\gamma \in \Gamma} \gamma_{1}^{i-1} \gamma_{2}^{j}=\sum_{k=1}^{n} e^{2 \pi \sqrt{-1} k\left(\left(l_{1} / n_{1}\right)(i-1)+\left(l_{2} / n_{2}\right) j\right)}$. This sum is $n$ if $\left(n / n_{1}\right) l_{1} i+\left(n / n_{2}\right) l_{2} j \equiv\left(n / n_{1}\right) l_{1}(\bmod n)$ and vanishes otherwise. Similarly for $u=2$.

When $X_{1}$ belongs to the Poincare domain and its eigenvalues are resonant, then the origin, $\lambda_{1}$ and $\lambda_{2}$ are colincar, besides $0 \notin\left[\lambda_{1}, \lambda_{2}\right]$, therefore there is only one possible type of resonance: $\lambda_{u}=m_{v} \lambda_{v}, u \neq v$.

When $X_{1}$ belongs to the Siegel domain the resonance $\lambda_{u}=m_{u} \lambda_{u}+$ $m_{v} \lambda_{v}$ generates an infinite family of resonanccs of type:

$$
\begin{array}{r}
\lambda_{u}=k\left(\left(m_{u}-1\right) \lambda_{u}+m_{v} \lambda_{v}\right)+\lambda_{u} \lambda_{v}=k\left(\left(m_{u}-1\right) \lambda_{u}+m_{v} \lambda_{v}\right)+\lambda_{v}, \\
k \in N, u \neq v .
\end{array}
$$

Finally let us make the following classification: 
Proposition 6. Let $\Gamma$ be a finite diagonal cyclic subgroup of $G L(2, \mathbf{C})$. The invariant normal forms are:

1. If $X_{1}$ belongs to the Poincaré domain.

$$
X\left(z_{1}, z_{2}\right)=X_{1}\left(z_{1}, z_{2}\right)+a_{i} z_{v}^{i} e_{u}
$$

where $\eta_{v} i \equiv \eta_{u}(\bmod n), u \neq v$ and $u, v=1,2$.

2. If $X_{1}$ belongs to the Siegel domain.

$$
X\left(z_{1}, z_{2}\right)=X_{1}\left(z_{1}, z_{2}\right)+\left(z_{1}\left(\sum_{k=1}^{\infty} a_{k} z_{1}^{k i} z_{2}^{k j}\right), z_{2}\left(\sum_{k=1}^{\infty} b_{k} z_{1}^{k i} z_{2}^{k j}\right)\right)
$$

where $\eta_{1} i \equiv-\eta_{2} j(\bmod n)$ or $k \equiv 0(\bmod n)$ and $i, j$ are like in proposition 5 .

For all cases if $l_{1} / n_{1}-l_{2} / n_{2} \notin Z$, then $X_{1}$ is diagonal.

This section can be applied to obtain conjugacies to normal forms in surfaces of type $\mathrm{C}^{2} / \Gamma$ where $\Gamma$ is a finite subgroup of $S U(2)$. These surfaces are embedded in $\mathbf{C}^{3}$ with an isolated singularity at the origin [KLE]. If $\Gamma$ is diagonal, then it is cyclic and generated by

$$
\left(\begin{array}{cc}
e^{2 \pi \sqrt{-1} / n} & 0 \\
0 & e^{-2 \pi \sqrt{-1} / n}
\end{array}\right)
$$

Proposition 6 applies in this case: $\eta_{1}=-\eta_{2}=1, l_{1} / n_{1}-l_{2} / n_{2}=$ $2 / n \notin \mathbf{Z}$ if $n \neq 2$.

When $\Gamma$ is non-diagonalizable, we have the groups which are the inverse image of the covering surjection $\rho: S U(2) \rightarrow S O(3)$ of the groups of index 2 (preserving orientation) of triangular spherical groups [MIL].

\section{References}

[BR] BRJuno, A. D., Analytic forms of Differential Equations, Trans. Moscow Math. Soc. 25 (1971), 131-282.

[CA] CARTan, H., "Quotient d'un espace analytique par un groupe d'automorphismes," Algebraic Geometry and Topology, Princeton U.P., 1957.

[CH] Chaperon, M., "Invariant manifolds and a preparation lemma for local holomorphic flows and actions," Holomorphic dynamics, Proceedings of Springer Verlag, Lecture Notes in Math. 1345, 1986. 
[DU] DuLAC, H., Solttions d'un systeme d'equations differentiellés dans le voisinage des valeurs singulières, Bull. Soc. Math. France 40 (1904).

[HU] HUMPHREYS, J., "Introduction to Lie algebras and representation theory," Springer Verlag.

[KLE] KLEIN, F., "Lectures on the Icosahedron and the solution of the equations of ffth degree," Teubner 1884, Dover, 1956.

[MIL] MiLnor, J., "On the 3-dimensional Brieskorn manifolds $M(p, q, r)$," Ann. of Math. Studies 84, Princeton U.P., 1975.

[SI] SIEGEL, C. L., "ber die normalform analytischer differentialgleinchungen in der Nhe einer gleichgewichtslosung," Nchr. Akad Wiss., Gottingen Math-Phys. k1, Math.-Phis.- Chem. Abt, 1952, pp. 21--30.

Instituto de Matemáticas

Universidad Nacional Autónoma de México

Ciudad Universitaria.

México 04510

D.F. MÉXICO

Primera versió rebuda el 30 de Gener de 1992, darrera versió rebuda el 13 d'Octubre de 1992 